\title{
Community-acquired Achromobacter xylosoxidans infection presenting as a cavitary lung disease in an immunocompetent patient
}

\author{
Chan Hee Hwang ${ }^{1}$, Woo Jin $\mathrm{Kim}^{2}$, Hye Young Jwa ${ }^{3}$, Sung Heon Song ${ }^{3}$ \\ ${ }^{1}$ Republic of Korea Defence Intelligence Command, Anyang, Korea \\ ${ }^{2}$ Department of Laboratory Medicine, Cheju Halla General Hospital, Jeju, Korea \\ ${ }^{3}$ Department of Internal Medicine, Cheju Halla General Hospital, Jeju, Korea
}

Received: May 14, 2019

Revised: July 26, 2019

Accepted: August 2, 2019

Corresponding author:

Sung Heon Song

Department of Internal Medicine,

Cheju Halla General Hospital, 65

Doreongno, Jeju 63127, Korea

Tel: +82-64-740-5009

Fax: +82-64-743-3110

E-mail: songsh0605@gmail.com
Achromobacter xylosoxidans is a gram-negative bacterium that can oxidize xylose. It is commonly found in contaminated soil and water but does not normally infect immunocompetent humans. We report a case of a cavitary lung lesion associated with community-acquired $A$. xylosoxidans infection, which mimicked pulmonary tuberculosis or lung cancer in an immunocompetent man. The patient was hospitalized due to hemoptysis, and chest computed tomography (CT) revealed a cavitary lesion in the superior segment of the left lower lobe. We performed bronchoscopy and bronchial washing, and subsequent bacterial cultures excluded pulmonary tuberculosis and identified A. xylosoxidans. We performed antibiotic sensitivity testing and treated the patient with a 6-week course of amoxicillin/clavulanate. After 2 months, follow-up chest CT revealed complete resolution of the cavitary lesion.

Keywords: Achromobacter xylosoxidans; Cavity; Hemoptysis; Immunocompetence; Pneumonia

\section{Introduction}

Achromobacter xylosoxidans is a gram-negative bacillus that can easily oxidize xylose. It is distributed in polluted environments (e.g., soil and water) and rarely infects human beings. However, A. xylosoxidans can opportunistically infect immune-deficient patients with various conditions, such as tumors, hematological disorders, organ transplantation, and acquired immune deficiency syndrome [1].

In Korea, cavitary lung lesions are typically observed in cases of lung cancer, pulmonary tuberculosis, bacterial pneumonia, and fungal lung infections. Furthermore, Yang et al. [2] reported that pulmonary tuberculosis is the most common cause of cavitary lung lesions, and most Korean clinicians primarily suspect pulmonary tuberculosis in these cases. Two cases of community-acquired A. xylosoxidans infection were reported by Lee et al. [3] in Korea. The patients were in the elderly age-group, and a single cavitary lung lesion was not observed in all patients [3]. Moreover, in our Korean medical literature search for cases of community-acquired A. xylosoxidans infection presenting as a cavitary lung lesion in an immunocompetent patient, no cases were found. Therefore, we report a case of hemoptysis (without immunodeficiency or chronic disorders) in a middle-aged man who was hospitalized for a single cavitary lung lesion in the superior segment of the left lower lobe. We suspected pulmonary tuberculosis because of its prevalence in Korea but detected A. xylosoxidans in bacterial testing and successfully treated the patient with targeted antibiotics. 


\section{Case}

A 46-year-old man was hospitalized for hemoptysis, with no other symptoms, such as fever, weight loss, or thoracic wall pain. He had a smoking history of 30 pack-years but had never been diagnosed with immunodeficiency or chronic disorders and had not visited a medical institution for the past 12 months. The patient was an office worker, but he went camping. Chest radiography revealed a cavitary lung lesion in the left lung (Fig. 1), and chest computed tomography (CT) revealed a smooth internal margin of a cavitary mass (approximate size, $2.4 \mathrm{~cm}$ ) with surrounding reticulonodular opacities in the superior segment of the left lower lobe. We did not detect mediastinal lymph node enlargement or pleural effusion (Fig. 2).

Laboratory testing revealed a white blood cell count of 12,690/ $\mathrm{mm}^{3}$ (range, 3,900-11,000/ $\mathrm{mm}^{3}$ ), hemoglobin level of $15.4 \mathrm{~g} / \mathrm{dL}$ (range, $13-18 \mathrm{~g} / \mathrm{dL}$ ), platelet count of $275,000 / \mathrm{mm}^{3}$ (range, $140,000-440,000 / \mathrm{mm}^{3}$ ), and C-reactive protein level of $4.40 \mathrm{mg} /$ $\mathrm{dL}$ (range, $\leq 0.3 \mathrm{mg} / \mathrm{dL}$ ). Biochemical testing revealed a total protein level of $7.1 \mathrm{~g} / \mathrm{dL}$ (range, 6.6-8.8 g/dL), albumin level of $4.3 \mathrm{~g} /$ $\mathrm{dL}$ (range, 3.5-5.2 g/dL), aspartate transaminase level of $32 \mathrm{IU} / \mathrm{L}$ (range, 0-40 IU/L), alanine transaminase level of $34 \mathrm{IU} / \mathrm{L}$ (range, 0-41 IU/L), blood urea nitrogen level of $10 \mathrm{mg} / \mathrm{dL}$ (range, 6-20 $\mathrm{mg} / \mathrm{dL}$ ), and creatinine level of $1.16 \mathrm{mg} / \mathrm{dL}$ (range, $0.7-1.2 \mathrm{mg} /$ dL). Immunological testing was negative for human immunodeficiency virus. We performed bronchoscopy to detect the lesion and

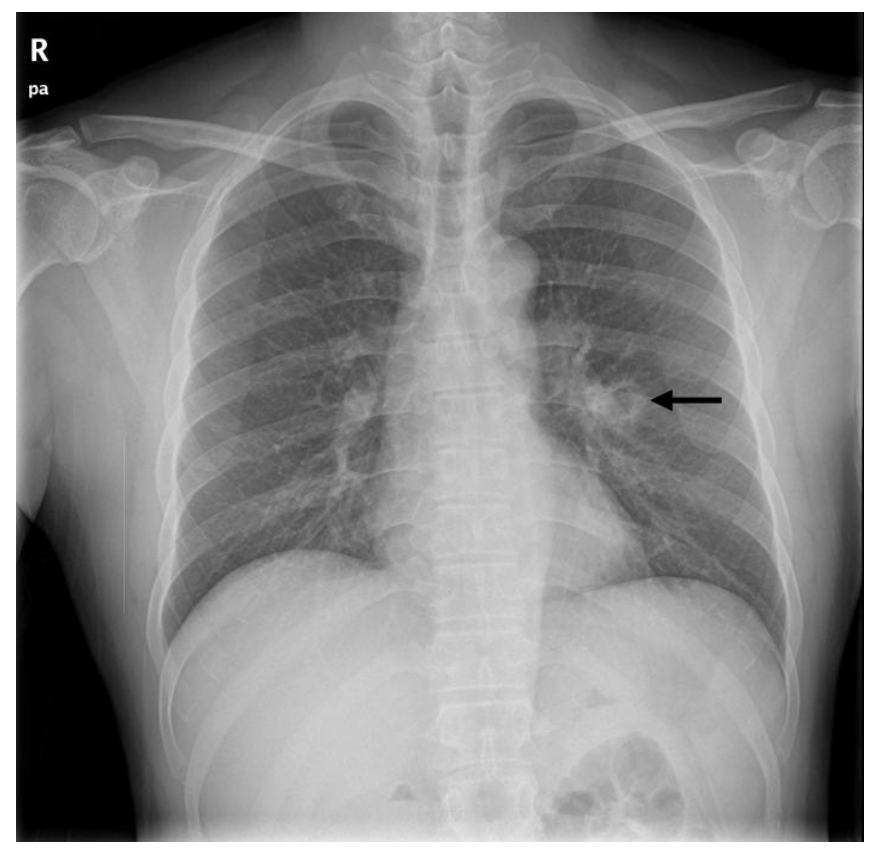

Fig. 1. Chest radiography reveals a cavitary lesion (arrow) in the left lung. obtain a specimen. Bronchoscopy revealed mild stenosis due to mucosal edematous changes in the lateralis bronchus orifice of the superior segment of the left lower lobe (Fig. 3). We attempted bronchial washing with a physiological salt solution. The acid-fast bacilli testing and cytopathological testing of the wash specimen revealed no specific findings, with negative polymerase chain reaction results for Mycobacterium tuberculosis and nontuberculous mycobacteria.

However, gram-negative identification of the wash specimen using Vitek ${ }^{\circledast} 2$ (bioMérieux, Marcy-I’Etoile, France) revealed the presence of gram-negative A. xylosoxidans. Therefore, we per-

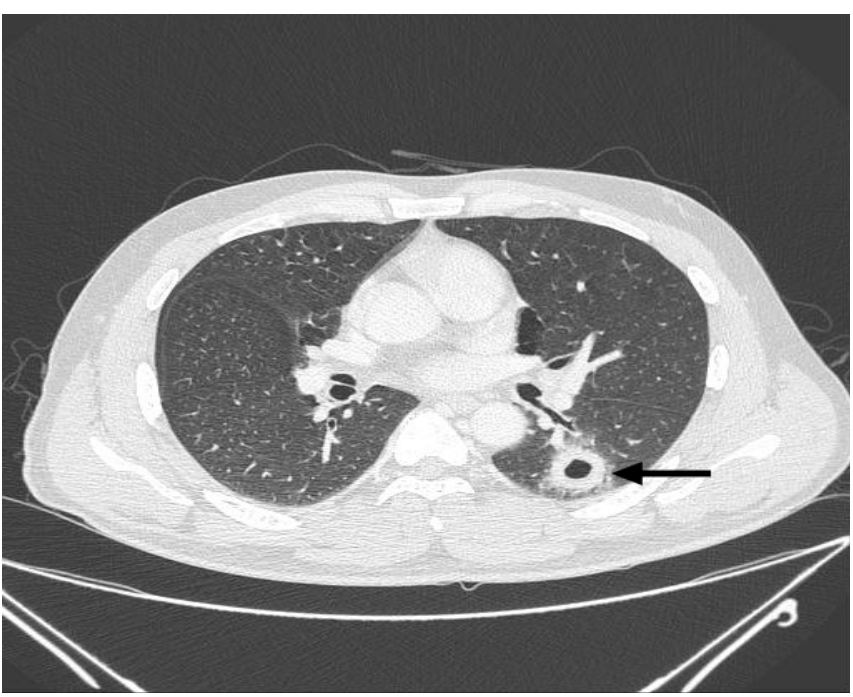

Fig. 2. Chest computed tomography reveals a smooth internal margin of a cavitary mass (approximate size, $2.4 \mathrm{~cm}$ ) with surrounding reticulonodular opacities (arrow) in the superior segment of the left lower lobe.

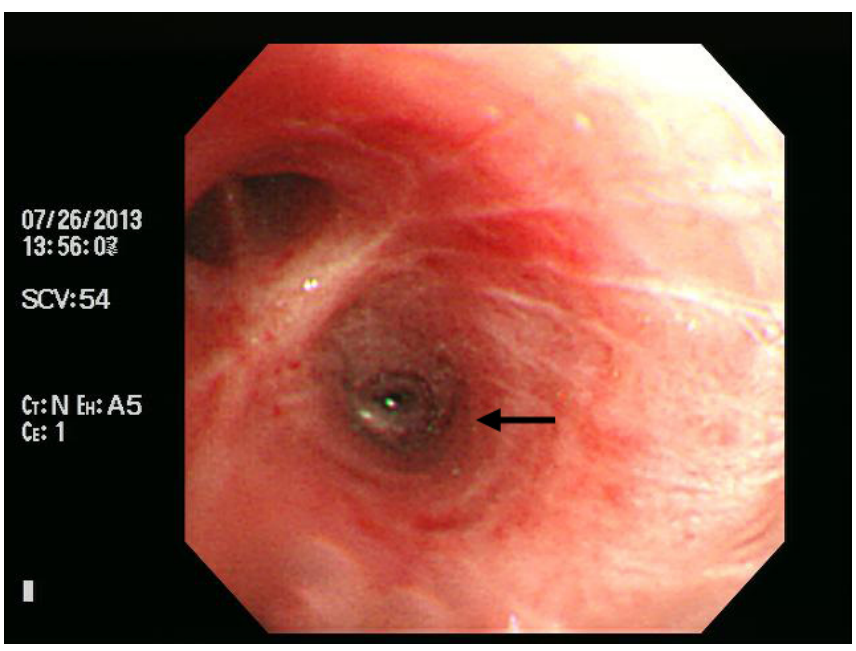

Fig. 3. Bronchoscopy reveals mild stenosis (arrow) in the lateralis bronchus orifice of the superior segment of the left lower lobe. 
formed antibiotic sensitivity testing based on the Clinical \& Laboratory Standards Institute criteria, which revealed sensitivity to amoxicillin/clavulanate, piperacillin/tazobactam, ceftazidime, imipenem, and trimethoprim/sulfamethoxazole and resistance to the other tested antibiotics (Table 1). We administered a 6-week outpatient course of amoxicillin/clavulanate. Follow-up chest CT at 2 months revealed no cavitary lung lesions (Fig. 4). In addition, the acid-fast bacilli testing of the follow-up bronchial wash specimen

Table 1. Antibiotic sensitivity test results

\begin{tabular}{lcc}
\hline \multirow{2}{*}{ Antibiotic } & \multicolumn{2}{c}{ Sputum culture } \\
\cline { 2 - 3 } & MIC & Interpretation \\
\hline Ampicillin & 16 & $\mathrm{I}$ \\
Amoxicillin/clavulanic acid & 8 & $\mathrm{~S}$ \\
Piperacillin/tazobactam & $\leq 4$ & $\mathrm{~S}$ \\
Cefazolin & $\geq 64$ & $\mathrm{R}$ \\
Cefoxitin & $\geq 64$ & $\mathrm{R}$ \\
Cefotaxime & $\geq 64$ & $\mathrm{R}$ \\
Ceftazidime & 4 & $\mathrm{~S}$ \\
Cefepime & $\geq 64$ & $\mathrm{R}$ \\
Aztreonam & $\geq 64$ & $\mathrm{R}$ \\
Imipenem & 1 & $\mathrm{~S}$ \\
Amikacin & $\geq 64$ & $\mathrm{R}$ \\
Gentamicin & $\geq 16$ & $\mathrm{R}$ \\
Ciprofloxacin & $\geq 4$ & $\mathrm{R}$ \\
Tigecycline & $\geq 8$ & $\mathrm{R}$ \\
Trimethoprim/sulfamethoaxazole & $\leq 20$ & $\mathrm{~S}$
\end{tabular}

MIC, minimum inhibitory concentration; I, intermediate; $S$, sensitive; $R$, resistant.

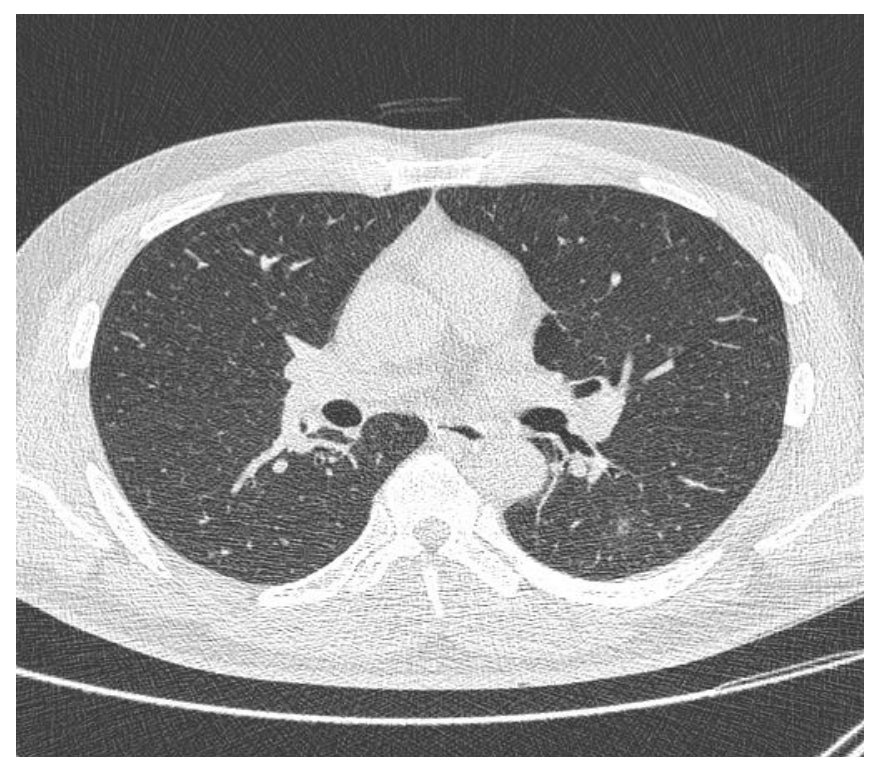

Fig. 4. Chest computed tomography after 2 months reveals complete resolution of the cavitary lesion in the superior segment of the left lower lobe. did not detect $M$. tuberculosis or nontuberculous mycobacteria.

\section{Discussion}

A. xylosoxidans was described by Yabuuchi and Oyama [4] in 1971 after isolation from ear discharge in patients with chronic otitis media and was included in the Alcaligenaceae family. It is an aerobic, motile, non-fermenting, gram-negative bacillus, which is catalase- and oxidase-positive. It is similar to the strains of Pseudomonas but has peritrichous rods [1]. It is ubiquitous in aqueous environments and has been found in well water, tap water, and swimming pools. It is a prevalent nosocomial colonizer that has been isolated from multiple aqueous solutions used in healthcare settings, such as non-bacteriostatic saline, dialysis solutions, contact lens solutions, CT contrast solutions, ultrasound gel, and chlorhexidine gluconate solutions. Moreover, it colonizes fomites, such as mechanical ventilators, neonatal incubators, intravenous catheters, epidural catheters, and urinary catheters [5].A. xylosoxidans infection typically presents with non-specific symptoms, and the bacteria have been isolated from multiple sources in patients, including blood, cerebrospinal fluid, stool, urine, sputum, skin, ear discharge, wounds, abscesses, bones, joints, endocardium, ascites fluid, corneal scrapings, and vitreous humor fluid $[1,5]$. However, A. xylosoxidans generally has low virulence and is typically isolated in cases of hospital-acquired infections that affect patients with hematological or oncological disorders (30\%), cardiac diseases $(21 \%)$, or immunosuppression (27\%) [5]. In the United States of America, A. xylosoxidans infection is relatively common among patients with cystic fibrosis (CF) [6], and recently, attention has been focused on A. xylosoxidans and Pseudomonas as the etiological agent in cases of chronic respiratory inflammation. In patients with $\mathrm{CF}, A$. xylosoxidans is detected in approximately $5.3 \%$ of the cases, and misdiagnosis is common because of its resemblance to Pseudomonas. Therefore, accurate bacterial and antibiotic sensitivity testing are important for identification and adequate treatment [6,7].

In adults, cavitary lung diseases are typically associated with malignant tumors and infective disorders $[2,8,9]$, and the disorder can be differentiated based on the lesion's thickness and location, characteristics of the inner margin, and patient's age. For example, cavity-forming carcinoma commonly has a smooth or irregular inner margin, and cavity-forming abscesses commonly have a rough inner margin. Furthermore, approximately $95 \%$ of the lesions with maximal wall thickness $>15 \mathrm{~mm}$ are malignant, and most lesions with maximal wall thickness $<4 \mathrm{~mm}$ are benign [8]. In the present case, the lesion exhibited a smooth inner margin, and the maximum thickness was $7.8 \mathrm{~mm}$. 
Tuberculosis is the predominant infectious cause of cavitary lung lesions in Korea and predominantly occurs in patients aged $<50$ years [8]. Furthermore, autoimmune and vascular disorders can cause cavitary lung diseases [2,9]. However, in patients with pulmonary tuberculosis, the presence of pulmonary cavitary lesions is associated with M. tuberculosis infection or reactivation. Moreover, the cavity's thickness is not useful in detecting pulmonary tuberculosis in cases of Mycobacteria infection, which can make the diagnosis difficult [2]. Therefore, bronchoscopy is typically needed to exclude malignancy (e.g., bronchial cancer or other bronchial diseases) in patients who are hospitalized for hemoptysis and have a smoking history [10]. This approach can also facilitate the immediate treatment of active bleeding, detection of intrabronchial lesions missed on radiological imaging (e.g., radiography or chest CT), and other testing (e.g., biopsy or bronchial washing). In the present case, the patient had a smoking history, which is a risk factor for malignancy; therefore, we performed chest CT and bronchoscopy to evaluate the cause of the hemoptysis. Chest CT revealed a smooth internal margin of a cavitary mass (approximate size, $2.4 \mathrm{~cm}$ ), suggesting pulmonary tuberculosis or malignancy, which are the most common causes of cavitary lung lesions in Korean men aged $<50$ years. However, culturing of the bronchial wash fluid revealed A. xylosoxidans.

Claassen et al. [5] experienced a similar case (73-year-old man with a smoking history of 30 pack-years and moderate chronic obstructive pulmonary disease) with lung lesions (maximum size, $13 \mathrm{~mm}$ ) and performed wedge resection because of the possibility of malignancy but isolated A. xylosoxidans from the surgical specimen. The authors provided 18 months of antibiotic treatment (levofloxacin, amoxicillin/clavulanate, doxycycline, and azithromycin), but lesion growth and multiplication led them to perform surgical resection [5]. Previous studies have indicated that A. xylosoxidans is resistant to aminoglycosides and rifampin but sensitive to imipenem, ceftazidime, $\beta$-lactamase inhibitor combinations, and trimethoprim/sulfamethoxazole. However, there are no accurate criteria for antibiotic sensitivity testing, and the existing data have been provided through a few in vivo experiments $[7,11]$. Nevertheless, the sensitivity results from the present case were similar to the results from previous experiments (Table 1).

In Korea, Lee at al. [3] reported 2 cases of pulmonary infection due to $A$. xylosoxidans in immunocompetent patients. One patient presented with a mass-like lesion suspected of lung cancer, and the other patient presented with pneumonia and para-pneumonic pleural effusion. In both cases, $A$. xylosoxidans was confirmed on culture of bronchial washing solution with bronchoscopy, as in our case. Our patient was relatively young and had a single cavitary lesion. The symptoms were clinically mild, and outpatient treatment was provided without hospitalization. However, the patients reported by Lee et al. [3] were the elderly and needed hospitalization due to relatively severe clinical conditions.

Infective disease or malignancy is typically suspected in cases of cavitary lung lesions detected using on radiography, and pulmonary tuberculosis testing is often performed in Korea (especially in cases of hemoptysis). However, these conditions must be differentiated based on the lesion's radiographic shape, size, and location; more extensive testing may be needed to confirm the shape of the lesion and specific disease (e.g., pulmonary tuberculosis or bacterial/fungal infection). Herein, we reported a 46-year-old man hospitalized for hemoptysis (with no history of chronic disorders or recent hospital visits). We suspected pulmonary tuberculosis, based on the lesion's radiographic shape and location, but culture of the bronchial wash fluid was negative for pulmonary tuberculosis and positive for $A$. xylosoxidans. Therefore, based on the antibiotic susceptibility results, we successfully treated the patient with an outpatient course of amoxicillin/ clavulanate.

\section{Acknowledgments}

\section{Conflicts of interest}

No potential conflict of interest relevant to this article were reported.

\section{ORCID}

Chan Hee Hwang, https://orcid.org/0000-0002-7892-1518

Woo Jin Kim, https://orcid.org/0000-0003-4999-1493

Hye Young Jwa, https://orcid.org/0000-0002-7485-811X

Sung Heon Song, https://orcid.org/0000-0002-9665-3369

\section{References}

1. Yabuuchi E, Yano I, Goto S, Tanimura E, Ito T, Ohyama A. Description of Achromobacter xylosoxidans Yabuuchi and Ohyama 1971. Int J Syst Bacteriol 1974;24:470-7.

2. Yang YW, Kang YA, Lee SH, Lee SM, Yoo CG, Kim YW, et al. Aetiologies and predictors of pulmonary cavities in South Korea. Int J Tuberc Lung Dis 2007;11:457-62.

3. Lee JH, Nam DH, Kim DH, Kim SH, Kim HN, Han CH, et al. Two cases of pulmonary infection due to A. xylosoxidans infection in an immunocompentent patient. Tuberc Respir Dis 2008;64:33-8.

4. Yabuuchi E, Oyama A. Achromobacter xylosoxidans n. sp. from human ear discharge. Jpn J Microbiol 1971;15:477-81.

5. Claassen SL, Reese JM, Mysliwiec V, Mahlen SD. Achromo- 
bacter xylosoxidans infection presenting as a pulmonary nodule mimicking cancer. J Clin Microbiol 2011;49:2751-4.

6. De Baets F, Schelstraete P, Van Daele S, Haerynck F, Vaneechoutte M. Achromobacter xylosoxidans in cystic fibrosis: prevalence and clinical relevance. J Cyst Fibros 2007;6:75-8.

7. Saiman L, Chen Y, Tabibi S, San Gabriel P, Zhou J, Liu Z, et al. Identification and antimicrobial susceptibility of Alcaligenes xylosoxidans isolated from patients with cystic fibrosis. J Clin Microbiol 2001;39:3942-5.

8. Kim NR, Han J. Pathologic review of cystic and cavitary lung diseases. Korean J Pathol 2012;46:407-14.

9. Gadkowski LB, Stout JE. Cavitary pulmonary disease. Clin Microbiol Rev 2008;21:305-33.

10. Bidwell JL, Pachner RW. Hemoptysis: diagnosis and management. Am Fam Physician 2005;72:1253-60.

11. Gómez-Cerezo J, Suárez I, Ríos JJ, Peña P, García de Miguel MJ, de José M, et al. Achromobacter xylosoxidans bacteremia: a 10year analysis of 54 cases. Eur J Clin Microbiol Infect Dis 2003;22:360-3. 